\title{
The transition to technology-enriched supported accommodation (TESA) for people living with dementia: the experience of formal carers
}

\author{
Janeet Rondon-Sulbaran ${ }^{1}$, Jean Daly Lynn², Brendan McCormack ${ }^{3}$, Assumpta Ryan ${ }^{4}$ \\ and Suzanne Martin ${ }^{2 \star}$ \\ ${ }^{1}$ Department of Management, Leadership \& Marketing, School of Health Sciences, Ulster University, \\ Jordanstown, UK, ${ }^{2}$ School of Health Sciences, Ulster University, Jordanstown, UK, ${ }^{3}$ Division of Nursing, \\ Occupational and Art Therapy, Queen Margaret University, Edinburgh, UK and ${ }^{4}$ School of Nursing, Ulster \\ University, Londonderry, UK \\ ${ }^{*}$ Corresponding author. Email: s.martin@ulster.ac.uk
}

(Accepted 24 April 2019; first published online 4 July 2019)

\begin{abstract}
This paper presents the experiences of formal carers working in technology-enriched supported accommodation for people living with dementia, examining their care-giving role from a person-centred care perspective. Within a qualitative study, 21 semi-structured interviews were conducted with formal carers and data were analysed following a thematic approach. Four main themes were identified that mapped to the attributes of the personcentred practice framework (PCPF): promoting choice and autonomy, staffing model, using assistive technology and feeling that 'you're doing a good job'. Central to personcentred practice in these settings was the promotion of choice, autonomy and independence. The dichotomy between safety and independence was evident, curtailing the opportunities within the environmental enablers and associated embedded assistive technologies. Formal carers reported considerable job satisfaction working in these settings. The small-scale, home-like facilities seemed to have a positive effect on job satisfaction. These findings are relevant to policy makers, commissioners and service providers, highlighting the facilitators of person-centred care in community dwellings for people living with dementia and the role of formal carers in promoting this approach.
\end{abstract}

Keywords: dementia care; formal carers; person-centred practice; supported accommodation; assistive technology

\section{Introduction}

Globally, dementia has become a significant public health challenge affecting more than 47 million people (World Health Organization (WHO), 2015). High prevalence rates of dementia have placed an enormous burden on the cost of dementia care across the world. Currently, in the United Kingdom (UK), there are 850,000

(C) Cambridge University Press 2019. This is an Open Access article, distributed under the terms of the Creative Commons Attribution licence (http://creativecommons.org/licenses/by/4.0/), which permits unrestricted re-use, distribution, and reproduction in any medium, provided the original work is properly cited. 
people living with dementia (Alzheimer's Society, 2014) with an estimated cost to the economy of $£ 26$ billion a year (Department of Health, 2015). Recent surveys have shown that 85 per cent of people diagnosed with dementia would prefer to stay at home for as long as possible rather than go into a care facility (Department of Health, 2015). Two-thirds of people with dementia in the UK live in the community and most care is provided by approximately 670,000 family and friends, saving the state over $£ 11$ billion per year (Alzheimer's Society, 2015).

While the provision of care at home, tailored to the needs of the person with dementia, their family and carers, has increased recently (Department of Health, 2015), many people living with dementia ultimately move into care homes when family carers are unable to manage their growing care needs (Livingston et al., 2017). In recent years, different models of long-term support have been introduced. For example, in the UK, extra-care housing or housing with care has emerged as an alternative to what was traditionally known as sheltered housing or very sheltered housing - a model known in the United States of America as assisted living (Brooker et al., 2011).

Similarly, there has been a growing interest in the development of small-scale, home-like residential care models with facilities specifically designed for people living with dementia incorporating technological innovation (Livingston et al., 2017). This approach to care for this population is an alternative to home and institutional care conceived to preserve the rights of the person providing choice and control over their housing options with a greater focus on independent living (Department of Health, 2015). However, little is known about the care strategies aligned with person-centred practice (PCP) that formal carers may or may not employ in their daily work in these facilities or the impact of assistive technologies on the workforce.

This study aimed to explore and understand the formal carers' experience working in a technology-enriched supported housing model. Throughout this study the term 'supported accommodation' rather than 'supported housing' was used, hence the acronym TESA (technology-enriched supported accommodation). Supported housing is defined as 'any housing scheme where housing is provided alongside care, support or supervision to help people live as independently as possible in the community ... Supported housing can provide long term support for years for some vulnerable groups such as older people' (Department for Communities and Local Government and Department for Work and Pensions, 2016: 9-10). The term technology-enriched is used as a descriptor for supported accommodation that has incorporated electronic assistive technology (e.g. sensor-based alarm alerts).

\section{Background}

PCP approaches to health care have been recognised and accepted as the best way to provide quality care, particularly for people living with dementia (Kitwood, 1997). Equally, in the UK, this approach has become an integral part of health and social care policy and strategies (Department of Health, 2009, 2015; Scottish Government, 2010; Department of Health, Social Care and Public Safety, Northern Ireland (DHSSPSNI), 2011a, 2011c; Welsh Government, 2017). PCP has permeated the long-term care sector and has become the central tenet of 
'culture change' targeted at the improvement of resident outcomes and the transformation of the facilities from medically driven institutions to individualised settings where care is provided according to the person's unique needs, choices, abilities and life history (Koren, 2010; McCormack and McCance, 2017a).

While some research highlights the benefits of supported accommodation for people living with dementia in terms of availability of opportunities for social interaction (Evans et al., 2007), little is known about the level of knowledge and ability of formal carers to support and preserve basic principles of PCP such as choice and dignity, particularly when assistive technology has been incorporated into the facilities. Research in this area is scarce. There is some evidence to suggest that the implementation of assistive technologies in this area is beset by various challenges. One of the challenges identified by Meiland et al. (2017) is related to the usability of technology by people experiencing dementia living in the community. Mainly, this concerns the following: (a) the relevance of the technology to specific needs; (b) the involvement of the person in the identification of needs; and (c) the management of ethical issues, such as loss of autonomy and privacy, and the difficulties in gaining informed consent when the person living with dementia does not understand or is unaware of the technology (Meiland et al., 2017).

There is a widespread consensus that the philosophy and principles of PCP should be at the heart of any existing and emerging models of dementia care (Koren, 2010; McCormack and McCance, 2017a). While there is evidence of the application of PCP approaches in long-term care settings, hospital wards and care homes (Williams et al., 2015; Fetherstonhaugh et al., 2016), such evidence has not yet been found in the supported housing model enriched with assistive technology. There is some evidence to suggest the positive impact of staff training aimed at dealing with psychiatric symptoms in care homes, as staff reported reduced symptoms in residents and changes in their own attitudes by adopting an empathetic approach that fostered the development of relationships (Goyder et al., 2012).

Other research suggests the need to redress the relations of power and change organisational cultures in dementia care in order to realise the potential contribution of direct-care staff in person-centred practice who feel disempowered and who receive limited training, recognition or remuneration (Scales et al., 2017). Similarly, other studies have highlighted the need to provide staff in long-term care settings and extra-care housing facilities with PCP training in order to enhance their acquired knowledge of the approach through their daily work experience and to support them in dealing with challenging behaviours (Evans et al., 2007; Hunter et al., 2016).

The theory of person centred care (PCC) in dementia first described by Kitwood (1997) postulates that the person is at the centre of their own care. The concept of personhood is at the core of PCC (Sabat and Harré, 1992; Kitwood, 1997; Rogers, 2011). A key challenge is that while the term PCC has become familiar in the field of dementia care, it has been challenging to agree on its components and meanings. For example, Brooker (2003) used the VIPS framework to describe PCC as a concept comprising four elements: $\mathrm{V}$ - valuing all people regardless of age and cognitive ability; I - recognising people as individuals; P - understanding the world from the perspective of the person living with dementia and carers; and $S$ - a positive social environment conducive to acceptable wellbeing. Additionally, McCormack 
and McCance $(2006,2010,2017 a)$ developed the person centred practice framework (PCPF) as a holistic structure that focuses on the characteristics of a personcentred culture within which PCC can be provided. This framework has been adopted in this study, as its holistic nature enables the analysis of a range of factors that might support person-centredness.

McCormack and McCance (2017b: 20) define person-centredness as

an approach to practice established through the formation and fostering of healthful relationships between all care providers, service users and others significant to them in their lives. It is underpinned by values of respect for persons, individual right to self-determination, mutual respect and understanding. It is enabled by cultures of empowerment that foster continuous approaches to practice development.

From this viewpoint, McCormack and McCance (2010, 2017b) have operationalised the factors that might enable person-centredness into a macro-context and four constructs: prerequisites, care environment, person-centred processes and person-centred outcomes. All constructs and their full set of attributes are shown in Figure 1.

\section{Methods}

The study was part of a larger qualitative investigation about the experience of living in TESA in which the views of people living with dementia as tenants in the facilities were sought in addition to the views of their informal (family/friend) and formal carers (paid care staff working in the facilities). This paper reports on the data obtained from formal carers.

\section{Aim}

The overall aim of this study was to explore formal carers' experiences of the transition by people living with dementia to TESA.

\section{Objectives}

The objectives were:

(1) To explore the extent to which the care practices of formal carers are person-centred in the context of alignment with the PCPF developed by McCormack and McCance (2017a).

(2) To explore the challenges associated with the promotion of PCP in supported accommodation.

(3) To explore the extent to which assistive technology hinders or supports person-centred practice.

(4) To make recommendations for policy practice and research.

\section{Setting and sample}

The study was conducted in all five Health and Social Care Trusts (HSCT) ${ }^{1}$ in a region of the UK where all facilities identified as providers of TESA for people 


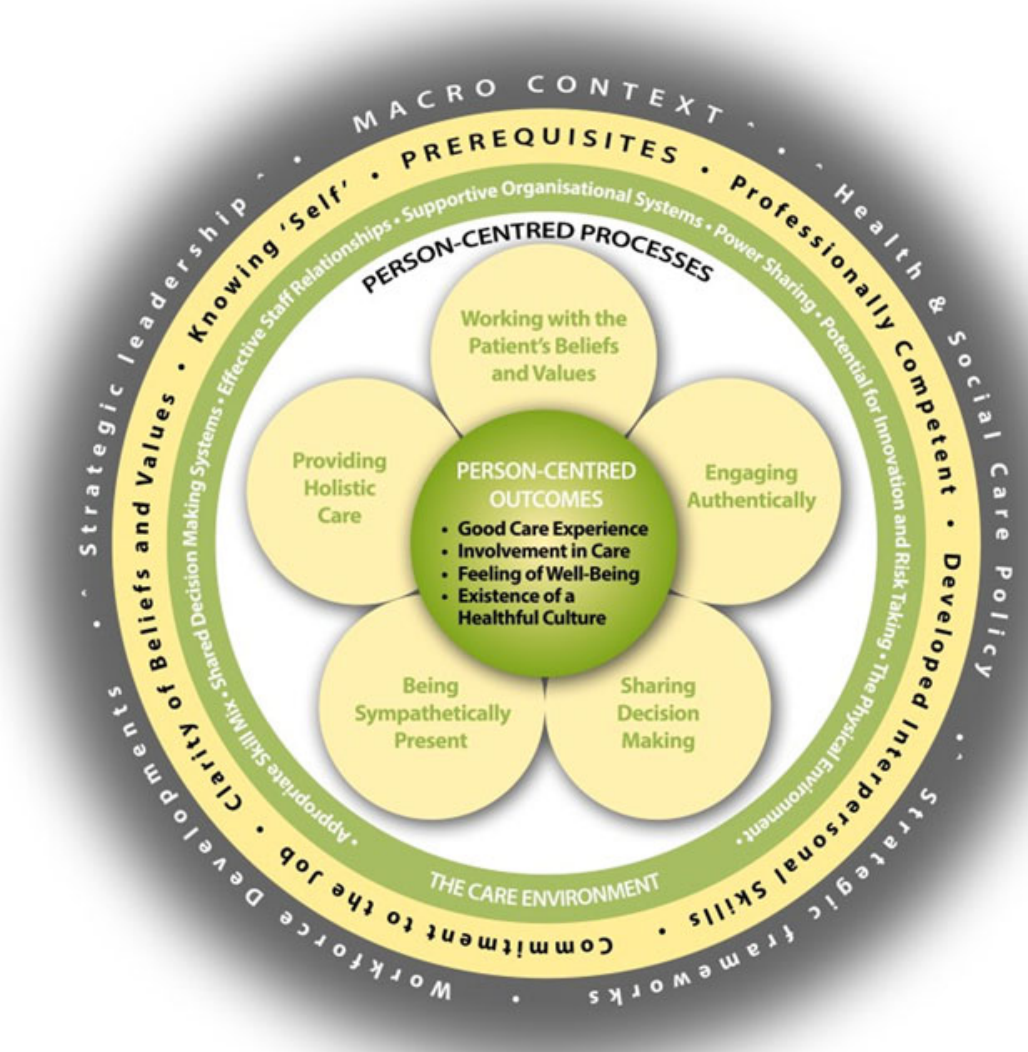

Figure 1. The person-centred practice framework.

living with dementia were invited to take part in the study. Participants were a purposive sample of formal carers at all levels (but mainly support workers) ${ }^{2}$ working in these TESA facilities. Access to the sample was obtained through the Supporting People Programme - a government initiative created to provide a range of housing services for vulnerable adults (Northern Ireland Housing Executive, 2017). Thus, ten TESA facilities registered in this programme were invited to participate in the study and staff were asked to partake in semi-structured interviews. One facility declined the invitation as they were undergoing an organisational restructuring at the time.

In most cases the primary funder for the facility was the HSCT with an approximate split of funding of 60 per cent HSCT and 40 per cent Supporting People Programme. Care in the facilities is provided by a mix of HSCT staff and/or voluntary-sector organisations; management is facilitated by housing associations. In Northern Ireland, these facilities are regulated by the Regulation and Quality Improvement Authority (RQIA). This is an independent body established in 
2005 to regulate and inspect the quality and availability of health and social care services in this province of the UK (RQIA, 2016). Managers of these facilities are required by the RQIA and in accordance with relevant legislation and DHSSPS standards, to deliver PCC (DHSSPSNI, 2011b). There is no known specific measures, however, for how person-centredness is operationalised across services.

The dwellings participating in the study consisted of a range of housing options. They included: small units for up to 12 people with private en-suite bedrooms and communal living and kitchen areas, bigger units of the same type for more than 60 people, and self-contained bungalows or apartments ( 25 or 30 per facility) within a complex that also offers communal recreational spaces and gardens (for more detail, see Table 1). By February 2016, when interviews commenced, there were approximately 185 formal carers working in these facilities.

The intercom system connected to handsets was a standard technology used in all the facilities. The various technologies used across the facilities, as shown in Table 2, were categorised into three types: alarms, sensors and monitors.

\section{Recruitment}

The nine participating facilities were approached in December 2015 when formalised written consent with their managers was completed. These managers were asked to inform their staff about the research and make information packs available to them. These packs included an information sheet, a consent form and a selfaddressed envelope to return responses directly to the researchers. A total of 23 responses were returned and 21 participants were interviewed, including three managers. Of the two potential participants not interviewed, one ceased employment with the facility after the interview was arranged and the other one could not attend the interview as initially agreed and it was not possible to reschedule it for a later date.

All participants were assigned an identity code which consisted of a whole number $(1,2,3$, etc.) preceded by the initials 'FC' (formal carer) and a capital letter from ' $\mathrm{A}$ ' to ' $\mathrm{I}$ ' which corresponded to the identity code of the site where they worked.

\section{Data collection}

Data were collected from February until November 2016. In total 21 semistructured interviews were conducted, lasting between 40 and 60 minutes. On the day of the interview JR-S, the researcher, formalised consent and reminded the participants about confidentiality and anonymity and the right to terminate the interview at any time or not to answer all the questions. All the interviews were undertaken in the supported accommodation facility where the formal carers worked at a time convenient to them. A relatively loose topic guide was used to provide guidance during the conversation while giving participants the opportunity to describe their world richly (Kvale, 2009). The interviews explored what the participants perceived to be their role as formal carers working in supported accommodation for people living with dementia, how they felt about the delivery of care in the facility and the impact of assistive technology on their role. The themes of the topic guide were aligned with the four constructs of the PCPF (McCormack and 
Table 1. Capacity of each of the facilities across the five Health and Social Care Trusts (HSCT) in Northern Ireland and occupancy when the research was conducted

\begin{tabular}{lccc}
\hline HSCT & Facility & Capacity & Number of occupants (\% occupancy) \\
\hline Belfast & Site A & 35 & $30(86)$ \\
\hline & Site B & 30 & $29(97)$ \\
\hline South Eastern & Site C & 25 & $25(100)$ \\
\hline & Site D & 30 & $25(83)$ \\
\hline Southern & Site E & 23 & $23(100)$ \\
\hline Northern & Site F & 12 & $12(100)$ \\
\hline Western & Site G & 61 & $54(89)$ \\
\hline & Site H & 15 & $15(100)$ \\
\hline Total & Site I & 35 & $30(86)$ \\
\hline
\end{tabular}

Note: $\mathrm{N}=243$.

Table 2. Categorisation of technology devices used across the facilities

\begin{tabular}{lll}
\hline Alarms & \multicolumn{1}{c}{ Sensors } & Monitors \\
\hline - Fall alarm/detector & - Motion sensor & - Water-flow control \\
- Smoke alarm & - Inactivity sensor & - Cooker monitor \\
- Flood alarm & - Automatic/motion-sensitive & (turn-off device) \\
- Carbon monoxide detector & lights & \\
- Heat/temperature extreme & - Water temperature & \\
alarm & - Pressure sensor: bed & \\
- Gas detector & - Pressure sensor: chair \\
& - Pressure sensor: floor \\
& - Door sensonsor \\
& - Enuresis/continence sensor & \\
\end{tabular}

McCance, 2017a) in order to elicit strategies of PCPF applied by formal carers during their day-to-day work in the various facilities. The PCPF, as shown in Table 3, has provided the theoretical framework in order to assess through systematic inquiry whether person-centred practice is present in the TESA model.

\section{Data analysis}

All interviews were transcribed verbatim by the researcher and also a professional transcriber. After checking the transcripts for discrepancies, data were anonymised and all identifiers were removed. Then data were uploaded on to NVivo 11, a software package for qualitative data analysis (Bazeley, 2013), and were analysed using a thematic approach. Themes or patterns were identified in a deductive or 'top-down' 
Table 3. Person-centred practice framework constructs

The macro-context includes major external factors related to health and social care policy, strategic frameworks, workforce developments and strategic leadership:

(1) Prerequisites focus on the attributes of the care worker and include: being professionally competent; having developed interpersonal skills; being committed to the job; being able to demonstrate clarity of beliefs and values; and knowing self.

(2) The care environment focuses on the context in which care is delivered and includes: appropriate skill mix; shared decision-making systems; effective staff relationships; supportive organisational systems; power sharing; potential for innovation and risk taking; and the physical environment.

(3) Person-centred processes focus on delivering care through a range of activities and include: working with patient's beliefs and values; engaging authentically; being sympathetically present; sharing decision-making; and providing holistic care.

(4) Person-centred outcomes, the central component of the framework, are the results of effective person-centred practice and include: good care experiences; involvement in care; feeling of wellbeing; and fostering a healthful culture.

Source: McCormack and McCance (2017a).

manner (Braun and Clarke, 2006). Through this process an initial list of codes was developed from the themes of the topic guide (Saldana, 2016); the latter were loosely aligned with the four constructs of the PCPF (McCormack and McCance, 2017a). This “"theoretical” thematic analysis' (Braun and Clarke, 2006: 84) helped to guide the analysis on to those aspects of the data particularly related to the constructs of the PCPF (McCormack and McCance, 2017a). Two researchers, separately, scrutinised the codes for internal reliability (LeCompte and Goetz, 1982). Competing and complementary themes were distinguished, allowing the researcher to agree on the four themes and corresponding sub-themes described in the findings. The outcome of this process, showing a list of the most illustrative codes generated in the initial themes that led to the final four themes, is presented in Figure 2.

\section{Findings}

Within the 21 participants interviewed, 17 were females and four males. The age range of participants varied between 18 and 69 years. The majority of participants were support workers (for details, see Table 4).

Exploration of the data revealed that aspects of the PCPF (McCormack and McCance, 2017a), mainly in the constructs of the care environment, the personcentred processes and outcomes, were reflected in the data. Four key main themes emerged from the data: (a) promoting choice and autonomy; (b) staffing model; (c) using assistive technology; and (d) feeling that 'you're doing a good job'.

\section{Promoting choice and autonomy}

At all sites there were numerous examples where the promotion of choice and autonomy was a key element of the staff approach to care. These examples varied from valuing the person and giving them choices, through to building relationships, 


\begin{tabular}{|c|c|c|}
\hline Theme & Codes & Four main themes \\
\hline Care approach & $\begin{array}{l}\text { Choice and shared decision-making } \\
\text { Holistic care } \\
\text { Sympathetic presence } \\
\text { Engagement } \\
\text { Offering support }\end{array}$ & $\begin{array}{l}\text { Promoting choice and } \\
\text { independence }\end{array}$ \\
\hline Care environment & $\begin{array}{l}\text { Skills and training } \\
\text { Staff relationships } \\
\text { Staff communication } \\
\text { Support from colleagues } \\
\text { Learning } \\
\text { Prevention } \\
\text { The physical environment }\end{array}$ & Staffing model \\
\hline Technology & $\begin{array}{l}\text { Non-intrusive } \\
\text { Prevention } \\
\text { Safety } \\
\text { Protection } \\
\text { Helping independence } \\
\text { Supporting staff }\end{array}$ & $\begin{array}{l}\text { Using assistive } \\
\text { technology }\end{array}$ \\
\hline Advantages for staff & $\begin{array}{l}\text { 'you're doing a good job' } \\
\text { Job satisfaction } \\
\text { Supporting health } \\
\text { Supporting wellbeing } \\
\text { Freeing time }\end{array}$ & $\begin{array}{l}\text { Feeling that 'you're } \\
\text { doing a good job' }\end{array}$ \\
\hline
\end{tabular}

Figure 2. Initial codes and themes leading to four main themes. 
Table 4. Demographic characteristics of participants

\begin{tabular}{|c|c|c|}
\hline Characteristics & $\mathrm{N}$ & $\%$ \\
\hline \multicolumn{3}{|l|}{ Gender $(N=21)$ : } \\
\hline Female & 17 & 81 \\
\hline Male & 4 & 19 \\
\hline \multicolumn{3}{|l|}{ Age $(N=21)$ : } \\
\hline $18-29$ & 3 & 14 \\
\hline $30-39$ & 5 & 24 \\
\hline $40-49$ & 5 & 24 \\
\hline $50-59$ & 7 & 33 \\
\hline $60-69$ & 1 & 5 \\
\hline \multicolumn{3}{|l|}{ Position $(N=21)$ : } \\
\hline Support Worker & 13 & 62 \\
\hline Scheme Manager & 3 & 14 \\
\hline Senior Support Worker & 2 & 9.5 \\
\hline Activity Co-ordinator/Co-ordinator & 2 & 9.5 \\
\hline Team Leader & 1 & 5 \\
\hline \multicolumn{3}{|l|}{ Length in post $(N=20)$ : } \\
\hline $6-11$ months & 2 & 10 \\
\hline $1-5$ years & 11 & 55 \\
\hline $6-11$ years & 5 & 25 \\
\hline $12-13$ years & 2 & 10 \\
\hline \multicolumn{3}{|l|}{ Years of experience in care $(\mathrm{N}=18)$ : } \\
\hline $5-10$ & 4 & 22 \\
\hline $11-20$ & 11 & 61 \\
\hline $21-30$ & 1 & 6 \\
\hline $30+$ & 2 & 11 \\
\hline
\end{tabular}

maximising skills and independence, and providing opportunities for meaningful activities.

Staff mentioned that the facilities allowed tenants choice about with whom and when they interacted, prioritising the opportunity to enjoy the privacy of their own homes. Staff appreciated the fact that tenants were able to make choices about who they let into their homes and to whom they chatted or were friendly with. Staff mentioned that the daily routine visits to the tenants' home within the facilities offered opportunities to build relationships, but it was always left to the tenants' discretion to avail or not of these opportunities. For instance, this staff member described the type of interaction that could take place in one of the facilities where the tenants lived in self-contained flats and received daily calls by support staff: 
It's just that you have your own wee flat and you're self-contained but you have a lot of, you know, you've the help there, you have your own privacy the way you would have in your own home ... there's the support and the girls, the staff go in and out for a short space of time and do what they need to do. And then if they want to have a chat or sit down, they'll sit and have a chat with you, but if they don't, then just go out again. (FC-C1)

There was a general acknowledgement of respecting the tenants' choices in all contexts, particularly in relation to food, dressing and personal hygiene, as shown in this quote:

Nobody is being told: 'Right, well, you're going into a shower and you're being put into a shower.' It's not about that, they all have that choice. (FC-G1)

It was interesting to see how some of the participants who had worked in residential care were able to make comparisons between the two models and found themselves in awe at the rigidity of the residential care system where meal schedules and menus are pre-established by others without any consultation with the residents. One participant said:

I would find it very difficult to go back to residential where you're all sitting down at this one time and you're having this for your dinner and you have no choice. They're picking what they want to eat, their families, there's consultation with them about what food they're having, they're not having a set menu, you know what I mean? So, to me, you can't compare the two, you can't compare the two, definitely not. (FC-D1)

It was widely agreed among the participants that the aim of supported living for people living with dementia was to promote independence while maximising the skills of the person. Thus, tenants were able to do things for themselves and, with the right encouragement and support from the participants, they could maintain their skills and abilities for as long as possible. This was an attitude that was prevalent in the majority of interviews, well reflected in this quote:

...our ethos is more basically trying to promote as much independence with them as possible. So, being more, us standing back and giving them by that to promote, for them to be able to do the things for themselves, to try and keep their minds stimulated. (FC-C2)

Some participants suggested that while independent living was part and parcel of supported accommodation, it was also necessary to offer adequate support to be able to keep tenants safe. Therefore, in their view, the TESA model was the most adequate intervention to achieve that, as this member of staff said:

It's [supported accommodation] the future, people with dementia need to be able to live in their own homes, they need to be supported, they need to feel safe; they need to have, it's their right to live at home and be happy and to be supported. 
And all this technology and all this support staff and all the partnerships together makes it possible for them. (FC-A2)

Many of the participants made references about the fluctuations of the condition and how sometimes the tenants' needs increased, therefore, on occasions, staff may be assuming a brief caring role while still supporting the tenants, as explained here:

But, again, sometimes some of the tenants need more care, and less support. So, it's just try and get the balance right ... But when they're better we are very much so, we are encouraging them to support them to do as much as they can for themselves. It is more about supporting rather than doing for, supporting with, or prompting with, or assisting, rather than just doing it for them. (FC-E1)

Being able to move about or go out was recognised by many of the participants as an ability that was necessary to maintain for as long as possible, however, it was also important to consider the potential risks in doing so and assess all situations accordingly, as explained by this staff:

Some of the tenants just go out shopping, they go to church, they do everything that they would've done in their own home; but they're assessed to see how safe they're on the road. That's an important assessment because you don't want to, this is not, you know, this is just supported housing, so we want them to enjoy life and not to be feeling enclosed. (FC-C1)

In general, it was recognised that the tenants in the majority of the facilities were at different levels in their dementia and that their skills varied and in most cases they declined. However, there were examples in some of the facilities where tenants were able to develop new skills which were not only enjoyable, but also enriching and rewarding. For example, this participant talked about one lady who became a facilitator of her own class in the facility:

...we have one lady, and after tapping into and encouraging her to do some of the different arts, music, the different activities in relation to that, she actually now has become so empowered and so interested that she herself runs a mindful colouring class for other tenants. And she's thriving because it takes her time to organise it, it gives her purpose, she's sharing her skills with other people and these are new skills she's learned. (FC-A3)

\section{Staffing model}

The majority of participants felt that they played a key role in establishing linkages and relationships with the tenants and family members, which in turn benefited everyone in different ways. For example, they found themselves in the privileged position of gaining or exchanging key information about the tenants with family members and/or amongst themselves. These exchanges were important, both in terms of ensuring participants that they were providing good quality care and that tenants and families felt supported, as explained in this excerpt: 
Families are very appreciative of the care and support they [the tenants] get in here. They really do appreciate it, and, you know, we pick up on something we'll say [it] to the family ... And then, the families are very grateful, and they are very open as well, like ourselves too, engage with other services. We've never had an issue with anything like that. (FC-E1)

Some participants mentioned how the flexibility offered by the supported accommodation model to include the input of families in the care of the tenants appeared to give them not only the opportunity to establish relationships with families, but also to have more control over their work and free some of their time to focus their attention on other tenants with higher needs. Specifically, one staff member talked about families getting involved in hands-on care on an ad hoc basis at lunch time, helping their relatives with their food, which then allowed them to help those on their own who also needed more support at meal times:

I find this approach [supported accommodation] better because the families come in and if their relatives are having lunch, they sit with them and talk with them while they're having their lunch ... Or, um, we have a couple of residents where you have to feed them, and if the relatives come in at lunch time they'll say: 'Look, we'll do that.' And then, that leaves you free to work with someone else. So, it's not just staff that are involved in that patient's welfare or needs, it's their families as well. So it is very inclusive, so it is. (FC-H2)

In contrast with the staff empowerment mentioned above, others felt that their work was at times overly task-oriented or that they had to spend too much time on domestic chores or similar tasks that prevented them from engaging in more individualised care, as this example illustrates:

We have to do a lot of different things ... A lot of stupid stuff, like cleaning and laundry ... and checks, ... we have to do water temperatures up on our corridors; that can take time. You have to go into every room and run every hot tap and record that ... just to make sure they're [the tenants] not putting on the hot water and scolding themselves ... So, all that stuff kind of ... it consumes you, so that you don't have time to just spend with them, or have a chat or do something nice for them. (FC-G1)

The above quote may be related to staffing levels which appeared to be variable in the different facilities. It emerged that in those facilities with low numbers of tenants (between 12 and 25 people) staff felt that the staffing levels were adequate, and they were able to provide holistic care:

You know, I've worked in nursing homes, I've seen that, you know, the staffing level here is brilliant because we need the staffing level that we've got to do what we do. You know, if we didn't have the four or five staff on a day we couldn't take them out and why shouldn't they be taken out, you know, they're still fit to do so. (FC-F2) 
This was emphasised by some of the senior staff interviewed in the smaller facilities who were vocal in expressing how lucky they felt for having appropriate staff ratios that could allow them to implement a PCC approach, as noted in this quote:

...whilst here it's, touch wood, our staff ratio is very good and enables us, our model of care, it enables us to spend a lot of time with the tenants, and they appreciate it, in other care environments that isn't not always the case. (FC-F1)

Indeed, in bigger facilities (of between 30 and 60 tenants) staff felt that the low staff to tenant ratio diminished the opportunities to be able to provide the holistic care promoted in the facilities:

It's just, sometimes, you would like, if you ... maybe your staffing levels could be down a bit sometimes, you know, if there's someone off sick you can't fulfil the job the best that you would want to because you'd have more work on. (FC-A2)

Overall, participants felt that they were well equipped for the job. They mentioned the induction sessions and training as invaluable to enhance their skills and to help them understand the nature and principles of the person-centred approach in supported accommodation, which for many differed from previous work experiences in the care sector, as stated by this participant:

To me at the start, you know, the training that is given is beneficial to me because it's a model I haven't experienced before. It's like they have their own accommodation, they have their own flats here, whereas in the private sector it is not designed that way. Um, and, you know, the training, needed, I did need the training just to be in that zone where you have to be first safeguarding the tenants. Yeah, it's good learning and good experience, more experience for me to see how it works. (FC-A1)

Most participants felt they had a fairly good understanding of dementia issues but, most importantly, they felt competent in their understanding of the person at various levels and being attuned to their feelings and needs, including the social, emotional and psychological. This participant illustrated this by saying:

It [dementia] can just be so debilitating to a person, you know, and they just lose their senses that they're important and that they're an individual and if we can give them a bit of comfort and a bit of love, you know, and make them feel valued and wanted because they can be so confused. (FC-B2)

Other participants gave examples of how collegiality and mentoring enriched their practice when they were confronting difficult situations which required the ability to see beyond the person's behaviour, as explained here by this participant talking about the agitation of a particular tenant:

I think the staff work well together in dealing with situations. If I had an issue, I'd say: 'I find this particular ... resident very challenging' ... I would say maybe to the 
senior: 'She seems a bit agitated today. She says she's a wee bit sore. Maybe she's got a wee UTI [urinary tract infection] or something?' And the senior would say: 'Well, get a wee sample and I'll...' It could end up that that was what would trigger her agitation. (FC-F3)

\section{Using assistive technology}

In general, participants appeared to favour the environmental enhancement of the facilities with assistive technologies. For many, technology was essential to their job. Universally, they found the telephone system with activation of multiple alarms and sensors vital to their job as they could swiftly be guided to the appropriate course of action in response to the specific alerts received through the system. This is illustrated by this participant:

When it comes to ... say, the telephone ... if there's a bed sensor and they get up, that'll come through our phone ... We know that they're out of bed, we're made alert and we don't have to be in the room to know, you know. I feel more at ease in my job. (FC-F3)

Assistive technology appeared to have the dual role of providing safety, security or protection for the tenants and to make formal carers feel more relaxed or 'at ease' in their job, as pointed out in this quote.

Unanimously, staff reported that the different types of assistive technologies in the various facilities could be used to prevent falls or walking around or wandering, therefore, offering protection to both the tenant and the participants, as explained here:

To me it's [the technology] all protection; it protects the tenants. You know, the technology that we have, the protection that we know, without intruding on them. ... Door alarms, you get someone that would want to come out, a lock won't let them walk around; the door alarms will let you know that. So, yeah, it protects us and it protects them. (FC-A1)

In this sense, assistive technology had the advantage of being non-obtrusive, which was perceived by participants as beneficial to the tenants as they did not have the feeling that they were being constantly observed or monitored:

...the assistive technologies support the staff to enable them in order to do their job. But it also gives that, um, non-obtrusive cover for tenants that they are safe within the environment they live in. (FC-A3)

The support offered by assistive technologies on the reduction of risk and the protection of tenants from harm to themselves or others was a topic profusely discussed by the majority of participants. For example, assistive technology enabled formal carers to monitor the execution of simple tasks like making cups of tea or cooking snacks, so that accidents could be prevented while still allowing the person to do things for themselves when they wanted. This participant summed this up: 
...there was a lady in one of the flats, you know, she still maybe like to get up and try and make her own wee cups of tea and do wee things ... they're supported to make their own food, you know, they've had their meals, it's: 'Enjoy your meal, I'll be back later...' I go out and I isolate that electric, so she can't be harmed, she can't be in danger, all through the technology, like, you know. (FC-A2)

The large majority of participants suggested that assistive technology was beneficial to supporting tenants to live independently and helping them with day-to-day tasks. Technology allowed tenants to work at their own pace and avoided the constant presence of someone who may end up intervening and doing things for them unnecessarily. This participant illustrated this with the example of a person feeling confident to use the toilet by him- or herself:

You know, even their push buttons in bathrooms and stuff, they're right beside the toilet and they know to press that so I think it gives them confidence as well to live independently as much as they can, that they can go in and use the toilet by themselves ... We don't have to be there all the time ... it's about them being able to do the things without us constantly following them. (FC-F2)

Some participants referred to the speed of communication and the rapid response to potential risks or adverse incidents that were also possible with the use of assistive technologies. For many, as suggested in this example, the benefits of assistive technology outweighed its disadvantages:

I think the advantages outweigh the disadvantages very much so. I don't think the communication would be the same if we didn't have a certain technology, the handsets. (FC-G3)

In general, two concerns emerged regarding the use of assistive technologies. Firstly, it was important that technologies were not used in a restrictive way and that they were non-intrusive and implemented according to the person's needs, as explained by this manager:

We have to be careful that all parties involved, the residents, professionals and ourselves, are implementing those [technologies] for the benefit of the client and practices cannot be in any way restrictive. There's emphasis on the word 'assistive'; it's to assist them in their day-to-day and to enhance whatever they have. So, we would carefully consider it before we put any assistive technology in place. (FC-H1)

Secondly, that assistive technologies did not replace the human contact that staff and tenants should enjoy in the caring relationship. In this sense, technologies were viewed as an ally that could allow more free time to formal carers to be able to engage in personalised activities with the tenants and release them from the constraints of task-oriented routines. As this staff described it, everyone can just get on 'living life':

You know, we don't have to be there all the time with them [the tenants] because that's not what it's about, you know. It's about them being able to do the things 
without us constantly following them ... So, if there is a couple of residents in bed, it means, say in the afternoon because there is a couple like to lie down in the afternoon after their dinner ... it means, we don't have to stay in the room with them or sit outside. We can be in the close area with other residents doing something, but we know if they get up off that bed, the bed sensor will go off and we can go straight away to them. So, it helps everybody because you're not neglecting anyone then which is a good thing, everybody's getting the same attention and just living life. (FC-F2)

\section{Feeling that 'you're doing a good job'}

For many of the participants the sense of 'doing a good job' was the main aspect of their work that kept them motivated and gave them immense job satisfaction. 'Doing a good job' consisted of various things. For some of the participants this was having an understanding of the person beyond their illness and doing nice things for them, as explained by this participant:

I always think, it must be so strange to have something on your mind or to feel something but not be able to say how you feel or what's bothering you, so ... if you could do one nice thing a day, I think if you can walk out that door and think: 'Oh, I made that person laugh' or 'I helped them...' or 'I changed their mood...' or something, definitely you satisfy yourself in the job. (FC-G3)

Other participants referred to the satisfaction they felt when tenants showed their appreciation for the 'little things' they did for them which could be making a cup of tea or helping them choose what to wear. For others, it was important to provide comfort, such as the one gained from a good night's sleep:

Seeing that nobody is unsettled. I always look that if you're on my shift and you got everybody organised and you got them into bed and you didn't have people ringing all night: you'd done a good job, you've got people well settled, they're comfortable and they've slept all night. That's good satisfaction. (FC-D1)

For a few participants, job satisfaction also derived from the belief that they were improving the quality of life of both families and tenants through the provision of adequate support that was going to keep the person experiencing dementia living independently for longer and even perhaps preventing him or her from a premature entry into nursing care. As this participant explained:

I just love this job. I think it's just a nice job. It's nice to give the family and the tenant a bit of support. It's nice to see them living their lives. It's nice to see them not having to go into a nursing home or anything like that. It's so nice to see they have their independence. (FC-C3)

\section{Discussion}

This study of TESA for people living with dementia has offered insights on the experiences of formal carers working in these facilities, ranging from small 
dwellings for up to 12 people with shared communal areas to medium-sized facilities providing self-contained independent accommodation for 30-60 people. The findings identified four main themes of dementia care in these facilities: 'promoting choice and autonomy', 'staffing model', 'using assistive technology' and 'feeling that "you're doing a good job"'. These themes largely mapped to the attributes of PCP contained in the PCPF proposed by McCormack and McCance (2017a). The attributes most prominently represented in these findings are those associated with the 'care environment', 'person-centred processes' and 'outcomes'.

These findings suggest that PCP is prevalent in these facilities. The emphasis on the promotion of choice, independence and autonomy reflects how the concept of personhood central to PCC (Sabat and Harré, 1992; Kitwood, 1997; Rogers, 2011) is embedded in the ethos of the facilities and demonstrates a culture change veered towards the adoption of person-centred processes, that is, the provision of holistic care and the authentic engagement of the person living with dementia whose beliefs, values, wishes and desires should be always recognised (McCormack and McCance, 2017a). These findings are substantiated by other research, as reported by Brownie and Nancarrow (2013) in their systematic review of the effects of PCP on residents and staff in care settings where in purpose-built, small-scale, home-like settings traditional nursing models have been dismantled and the decision-making on care delivery has shifted to the residents. It will be important to learn more about the mix of tenants in these facilities, in terms of the severity of the dementia, in order to facilitate the right conditions for formal carers, as they found that the heterogeneity of needs impacted on the strategies to support independence and maintenance or maximisation of skills. Specifically, many of the staff referred to safety issues and potential risks which needed to be monitored and assessed to strike the right balance between safety and independence, and avoid the dilemmas posed between 'too much intervention' and 'too much risk' (Molony and Bouma, 2013: 80). The key issue of independence for people living with dementia in supported housing schemes has been highlighted by other authors (Evans et al., 2007), and consonant with their findings, we have revealed that with the appropriate support from formal carers, tenants in these facilities are given choice and autonomy in their lives to make decisions about simple things like what to wear or what to eat, or what to do or not to do, and by doing so are treated with dignity and respect.

This care delivery focused on engagement, authenticity, shared decision-making and holistic care is supported by the appropriate conditions of the care environment. While it was evident that in the smaller facilities of up to 25 tenants, formal carers were able to focus more on aspects of care most commonly associated with PCC practices and felt competent and empowered, those working in bigger facilities mentioned the problems they face when their time is consumed by task-oriented duties which prevent them from practising a more individualised humanistic approach to care (Rasin and Kautz, 2007; Brownie and Nancarrow, 2013; Sjogren et al., 2017). This is a very important finding in our study given that the facilities have the added environmental enhancement which has been associated with small-scale, home-like environments proven to be successful in implementing PCC that has been highly underpinned by culture change models (Verbeek 
et al., 2012; Li and Porock, 2014). Formal carers felt competent and confident with the assistive technology available in the facilities.

There was also evidence that assistive technology supported various aspects of PCP, however, it also appeared that the technologies could have the purpose of an extra 'safety blanket' for the formal carers who may seem to sacrifice autonomy for the safety of the tenants, which differs from other studies in this area (Landau et al., 2010), and confirms other findings reporting reduced risk for tenants and reassurance to staff who feel more relaxed in their roles (Meiland et al., 2017). Other ethical issues identified concerned the extent to which tenants provided informed consent for the use of the technologies, specifically those used to monitor and alert staff. While managers emphasised the adoption of any assistive technology involving all parties (i.e. 'residents', professionals and the managers themselves) according to need and to enhance the quality of life of the tenants, it was not clear to what extent and at what stage of the dementia the tenant is aware of the situation and capable of making an informed decision. By no means is there an implication that assistive technology has been used unethically, however, there appears to be a gap in advanced care planning, so that the tenants' preferences and desires are considered when their capacity to make decisions has diminished (Livingston et al., 2017).

The feeling that 'you're doing a good job', reported as the fourth key theme in our study, related to the high levels of job satisfaction demonstrated by the participants. This is consonant with research that has confirmed the positive effects on staff job satisfaction with the provision of PCC/P (McCormack et al., 2010; Brownie and Nancarrow, 2013). While some formal carers expressed some concerns about the staffing levels, mainly in larger facilities (of between 30 and 60 tenants), there appeared to be a balanced staff to tenant ratio in small-scale facilities which contributed to increased opportunities to provide quality care and time to the tenants, which is confirmed by research undertaken elsewhere (Rasin and Kautz, 2007). Formal carers in this study felt that they could have a positive impact on the life of the people with dementia in the facilities by doing 'little things' for them and in return the carers derived enormous job satisfaction from the appreciation the tenants showed.

The new knowledge derived from this study in the use of PCPs confirms some of the evidence that the supported housing model could assist people living with dementia (Evans and Means, 2006). Through the accounts of the formal carers it has been shown that this model not only is being delivered using a PCC approach as operationalised by McCormack and McCance (2017a) in their PCPF, but also contains the added value of the environmental improvement, another feature identified by other authors as essential to PCP (Brownie and Nancarrow, 2013). This is important for policy makers and service commissioners and for the future role of the voluntary sector and formal carers and other professionals involved in the delivery of care for people living with dementia. It appears that careful consideration should be given to find appropriate ways to disseminate good practice and the replication of those models of care, in particular the small-scale, home-like environments conducive to better outcomes for both the person living with dementia and the formal carers. There is no doubt there are limitations with this study. For example, recruitment within a single region of the UK, within which there is 
an integrated health and social care system, means that the experiences of staff may not be replicated in other areas. In addition, our limited time with staff to gather data could be considered a weakness, therefore adopting a more immersive methodology, e.g. ethnography, may prove insightful. Furthermore, whilst the PCPF was adopted as the overarching framework for this project, that in itself may have bound our thinking. Nonetheless, new insights have been gained and this is the first comprehensive study exploring novel technology-enriched supported housing.

\section{Conclusion}

This paper describes research, as part of a larger study, completed on the experience of formal carers of people with dementia who transition into TESA. The findings provide an understanding of the extent to which this practice has adopted a PCC approach. These findings are relevant from a health and social care point of view which emphasises the need to provide appropriate services based on a whole systems approach to care. The key themes highlighted here that characterise the person-centred practice in these facilities can be the precursors for further research focused on different social policy contexts important for understanding the differences in size variation that could overcome the barriers that seem to make PCP more successful in smaller dwellings.

Acknowledgements. Thanks are extended to the Health and Social Care (HSC) Research \& Development Office and Atlantic Philanthropies for funding this project. We are sincerely grateful to the many people living in TESA facilities within Northern Ireland who opened up their homes and lives to us. The team appreciates the openness and engagement of family and care staff with this project. Hopefully the voice of all can be heard within this work.

Financial support. This project was funded by the Health and Social Care (HSC) Research \& Development Division of the Public Health Agency in Northern Ireland (Reference COM/4955/14) in conjunction with the Atlantic Philanthropies, an international grant-awarding body. The project is part of the HSC research strand into the 'Care of People with Dementia in Northern Ireland'. None of the awarding bodies have participated in any of the activities related to the research.

Conflict of interest. The authors declare no conflicts of interest.

Ethical standards. This study was given a favourable ethical opinion by the Research Office of Ethics Committees of Northern Ireland (ORECNI) on 28 August 2015 under REC Reference 15/NI/0160.

\section{Notes}

1 In Northern Ireland, health and social care services are publicly funded. The Department of Health is responsible for its funding while the Public Health Agency is the executive body responsible for the delivery of health and social care services across Northern Ireland through its five HSCT. This service is free of charge to all citizens of Northern Ireland and the rest of the UK.

2 Support worker is defined as 'a person who is employed on an individual basis to foster independence and provide assistance for a service user in areas of ordinary life such as communication, employment, social participation and who may take on secondary tasks in respect of advocacy, personal care and learning'. In social care, support workers have been seen as employees without professional qualifications, although many employers do encourage staff to study for National Vocational Qualifications (NVQs) while at work (Manthorpe et al., 2010: 317). Here we use the term 'formal carers' rather than 'support workers' and we use the term 'tenant' rather than 'resident' when referring to the person living with dementia residing in supported accommodation in the community who are visited by 'formal carers'. 


\section{References}

Alzheimer's Society (2014) Dementia UK: Update. London: Alzheimer's Society.

Alzheimer's Society (2015) Dementia 2015: Aiming Higher to Transform Lives. London: Alzheimer's Society.

Bazeley P (2013) Qualitative Data Analysis with NVivo. Los Angeles, CA: Sage.

Braun V and Clarke V (2006) Using thematic analysis in psychology. Qualitative Research in Psychology 3, 77-101.

Brooker DJ, Argyle E, Scally AJ and Clancy D (2011) The Enriched Opportunities Programme for people with dementia: a cluster-randomised controlled trial in 10 extra care housing schemes. Aging \& Mental Health 8, 1008-1017.

Brooker D (2003) What is person-centred care in dementia? Reviews in Clinical Gerontology 3, 215-222.

Brownie S and Nancarrow S (2013) Effects of person-centered care on residents and staff in aged-care facilities: a systematic review. Clinical Interventions in Aging 8, 1-10.

Department for Communities and Local Government and Department for Work and Pensions (2016) Funding for Supported Housing: Consultation. London: Department for Communities and Local Government and Department for Work and Pensions.

Department of Health (2009) Living Well with Dementia. A National Dementia Strategy: Putting People First. Leeds, UK: Department of Health.

Department of Health (2015) Prime Minister's Challenge on Dementia 2020. London: Department of Health.

Department of Health, Social Care and Public Safety, Northern Ireland (DHSSPSNI) (2011a) Improving Dementia Services in Northern Ireland. A Regional Strategy. Belfast: DHSSPSNI.

Department of Health, Social Care and Public Safety, Northern Ireland (DHSSPSNI) (2011b) Residential Family Centres. Minimum Standards. Belfast: DHSSPSNI.

Department of Health, Social Care and Public Safety, Northern Ireland (DHSSPSNI) (2011c) Transforming Your Care: A Review of Health and Social Care in Northern Ireland. Belfast: DHSSPSNI.

Evans S, Fear T, Means R and Vallelly S (2007) Supporting independence for people with dementia in extra care housing. Dementia 1, 144-150.

Evans S and Means R (2006) Perspectives on risk for older people with dementia in extra care housing in the UK: findings from a longitudinal study. International Journal on Disability and Human Development $1,77-82$.

Fetherstonhaugh D, Tarzia L, Bauer M, Nay R and Beattie E (2016) 'The red dress or the blue?' Journal of Applied Gerontology 2, 209-226.

Goyder J, Orrell M, Wenborn J and Spector A (2012) Staff training using STAR: a pilot study in UK care homes. International Psychogeriatrics 6, 911-920.

Hunter P, Hadjistavropoulos T and Kaasalainen S (2016) A qualitative study of nursing assistants' awareness of person-centred approaches to dementia care. Ageing \& Society 6, 1211-1237.

Kitwood TM (1997) Dementia Reconsidered: The Person Comes First. Buckingham, UK: Open University Press.

Koren MJ (2010) Person-centered care for nursing home residents: the culture-change movement. Health Affairs (Project Hope) 2, 312-317.

Kvale S (2009) Interviews: Learning the Craft of Qualitative Research Interviewing. Thousand Oaks, CA: Sage.

Landau R, Auslander GK, Werner S, Shoval N and Heinik J (2010) Families' and professional caregivers' views of using advanced technology to track people with dementia. Qualitative Health Research 3, 409-419.

LeCompte MD and Goetz JP (1982) Problems of reliability and validity in ethnographic research. Review of Educational Research 1, 31-60.

Li J and Porock D (2014) Resident outcomes of person-centered care in long-term care: a narrative review of interventional research. International Journal of Nursing Studies 10, 1395-1415.

Livingston G, Sommerlad A, Orgeta V, Costafreda SG, Huntley J, Ames D, Ballard C, Banerjee S, Burns A, Cohen-Mansfield J, Cooper C, Fox N, Gitlin LN, Howard R, Kales HC, Larson EB, Ritchie K, Rockwood K, Sampson EL, Samus Q, Schneider LS, Selbæk G, Teri L and Mukadam N (2017) Dementia prevention, intervention, and care. The Lancet 390, 2673-2734. 
Manthorpe J, Martineau S, Moriarty J, Hussein S and Stevens M (2010) Support workers in social care in England: a scoping study. Health \& Social Care in the Community 3, 316-324.

McCormack B, Dewing J, Breslin L, Coyne-Nevin A, Kennedy K, Manning M, Peelo-Kilroe L, Tobin C and Slater P (2010) Developing person-centred practice: nursing outcomes arising from changes to the care environment in residential settings for older people. International Journal of Older People Nursing $\mathbf{2}$, 93-107.

McCormack B and McCance TV (2006) Development of a framework for person-centred nursing. Journal of Advanced Nursing 5, 472-479.

McCormack B and McCance T (2010) Person-centred Nursing: Theory and Practice. Oxford: Wiley-Blackwell.

McCormack B and McCance T (eds) (2017a) Person-centred Practice in Nursing and Health Care: Theory and Practice. Chichester, UK: Wiley Blackwell.

McCormack B and McCance T (2017b) Underpinning principles of person-centred practice. In McCormack B and McCance T (eds), Person-centred Practice in Nursing and Health Care: Theory and Practice. Chichester, UK: Wiley Blackwell, pp. 13-35.

Meiland F, Innes A, Mountain G, Robinson L, van der Roest H, Garcia-Casal JA, Gove D, Thyrian JR, Evans S, Droes RM, Kelly F, Kurz A, Casey D, Szczesniak D, Dening T, Craven MP, Span M, Felzmann H, Tsolaki M and Franco-Martin M (2017) Technologies to support community-dwelling persons with dementia: a position paper on issues regarding development, usability, effectiveness and cost-effectiveness, deployment, and ethics. JMIR Rehabilitation and Assistive Technologies 1, e1.

Molony SL and Bouma R (2013) The care manager role in person-centered care for people with dementia. Generations 3, 79-82.

Northern Ireland Housing Executive (2017) The Supporting People Programme. Available at https://www. nihe.gov.uk/index/corporate/supporting_people_programme.htm.

Rasin J and Kautz DD (2007) Knowing the resident with dementia: perspectives of assisted living facility caregivers. Journal of Gerontological Nursing 9, 30-36.

Regulation and Quality Improvement Authority (RQIA) (2016) About Us. Assurance, Challenge and Improvement in Health and Social Care. Belfast: RQIA.

Rogers CR (2011) On Becoming a Person: A Therapists's View of Psychotherapy. London: Constable \& Robinson.

Sabat SR and Harré R (1992) The construction and deconstruction of self in Alzheimer's disease. Ageing \& Society 4, 443-461.

Saldana J (2016) The Coding Manual for Qualitative Researchers. Los Angeles, CA: Sage.

Scales K, Bailey S, Middleton J and Schneider J (2017) Power, empowerment, and person-centred care: using ethnography to examine the everyday practice of unregistered dementia care staff. Sociology of Health and Illness 2, 227-343.

Scottish Government (2010) Scotland's National Dementia Strategy. Edinburgh: Healthier Scotland, Scottish Government.

Sjogren K, Lindkvist M, Sandman PO, Zingmark K and Edvardsson D (2017) Organisational and environmental characteristics of residential aged care units providing highly person-centred care: a cross sectional study. BMC Nursing 44, 017-0240-4, eCollection 2017.

Verbeek H, Zwakhalen SM, van Rossum E, Kempen GI and Hamers JP (2012) Small-scale, homelike facilities in dementia care: a process evaluation into the experiences of family caregivers and nursing staff. International Journal of Nursing Studies 1, 21-29.

Welsh Government (2017) Draft Strategy: Together for a Dementia Friendly Wales. Cardiff: Mental Health and Vulnerable Groups Division, Welsh Government.

Williams J, Hadjistavropoulos T, Ghandehari O, Yao X and Lix L (2015) An evaluation of a personcentred care programme for long-term care facilities. Ageing \& Society 3, 457-488.

World Health Organization (2015) World Report on Ageing and Health. Geneva: World Health Organization.

Cite this article: Rondon-Sulbaran J, Daly Lynn J, McCormack B, Ryan A, Martin S (2020). The transition to technology-enriched supported accommodation (TESA) for people living with dementia: the experience of formal carers. Ageing \& Society 40, 2287-2308. https://doi.org/10.1017/S0144686X19000588 